\title{
A Pilot Exploration of Systematic Ideation Methods and Tools on Design Learning
}

\author{
Gül E. Okudan, Ming-Chuan Chiu and Chun-yu Lin \\ Penn State University \\ University Park, PA, USA \\ Noe Vargas Hernandez \\ University of Texas at El Paso \\ El Paso, TX, USA
}

\author{
Linda C. Schmidt \\ University of Maryland \\ College Park, MD, USA \\ Julie Linsey \\ Texas A\&M University \\ College Station, TX, USA
}

\begin{abstract}
Today, more than ever, we need engineering graduates equipped with effective design skills and a high degree of ideation competence. This paper presents results of our pilot study on improving the ideation performance of undergraduate engineers through training in TRIZ and emphasis on sketching. This is the first study of its type to rigorously test the value of TRIZ on ideation performance. Positive study findings would provide guidance to introductory and capstone design course instructors, as well industry practitioners who intend to enhance their ideation toolkits. Our objective is to test our hypotheses that TRIZ, or sketching emphasis improves design ideation alone or in combination. A series of classroom studies using investigatordeveloped interventions (modules) that train students in TRIZ and Sketching Importance is run. Class participants perform ideation exercises. The results of the ideation exercises are assessed and scored for Novelty, Variety and Quantity. In the paper, we document the results of the pilot experimentation. Overall, pilot studies will help fine tune the experimental approach.
\end{abstract}

Keywords - engineering design, ideation metrics

\section{INTRODUCTION}

The design ability of engineering students is built upon a foundation in three areas: 1) design process knowledge; 2) design analysis knowledge; and 3) creative processing ability, also called ideation. Design process knowledge, in general, is taught in first year design courses, and then practiced throughout the engineering curriculum culminating in the capstone design course. During second and third year courses, the engineering curriculum focuses on analytical concepts and techniques ultimately intended to support design analysis ability. Given the overcrowded traditional engineering curriculum, it is not surprising that students do not improve their creative processing skills.

Engineering design course instructors face a nearly insurmountable task: devise a combination of instructional events that will allow students to learn the best design methods possible and give them tasks that they can use to demonstrate their learned capability. Meeting this mark is not adequate anymore as instructors must lead students to learn to be creative in their designs and innovative in their applications so that they can become the next generation of successful engineers. Effective research on design learning will allow design theory and methodology researchers to make recommendations to improve design education practice. This research focuses on the impact of using creativity enhancing methods and tools to improve engineering students' design ideation process.

In this research, we propose to integrate TRIZ (a systematic problem solving approach) and sketching, and assess their effectiveness in support of ideation. In this integration, TRIZ will provide the systematic innovative problem solving method and sketching will externalize and enhance the design ideation.

\section{BACKGROUND}

Traditional approaches to creativity, which advocates using brainstorming, etc., call upon designers to look inward for inspiration, and then communicate their ideas to others to create a synergetic and shared experience. This work focuses on a method (TRIZ) and a practice (sketching) that provide additional external information and cognitive support to enhance the ideation process. TRIZ is a systematic approach to the generation of innovative designs to seemingly intractable problems. It was first developed in Russia by Genrich Altshuller after World War II and grew in prominence there in the early sixties and seventies. TRIZ is based on the analysis of hundreds of thousands of patents. These original analyses articulated numerous solution patterns from diverse disciplines. The patterns and the tools are continually being updated by researchers worldwide. TRIZ has been recognized as a concept generation process that can develop clever solutions to problems by using the condensed knowledge of thousands of past inventors. It provides steps that allow design teams to avoid the "psychological inertia" that tends to draw them to common, comfortable solutions when better, nontraditional ones may exist.

Research has shown that TRIZ has potential when used with brainstorming. For example, Okudan et al. [1] compared 
the ideation effectiveness of TRIZ and brainstorming in comparison to only using brainstorming measured by three ideation metrics: quantity, variety, and novelty as described by Shah et al. [2]. The design problem students solved was focused on air velocity control for fumehoods, and was presented by an industry sponsor. Results indicate significant gains in ideation metrics when TRIZ is used with brainstorming. However, the study was descriptive in nature, and does not explain why TRIZ is effective. Researchers in the UK found that introducing stimuli in the form of TRIZ innovative principles supported the rate of ideation generation during brainstorming sessions and lead to less obvious ideas [3]. Work with student groups concluded that the nature of the provided stimulus was important on its ideation impact. This study found that the more disparate the stimulus is, the more difficult it seems to be for designers to use it, thus the context within which the cues are provided is important for success [4]. Another study with engineering students focused on the impact of lexical stimuli (words) on design generation and found that students tended to use the words as verbs, especially when the stimulus was seemingly unconnected to the design task [5]. The same lexical study found that the dichotomous stimulus (like that presented in the TRIZ method) led to concepts that were judged by raters to be more novel than other concepts.

Sketching was a critical skill in traditional engineering design but the practice has become less important to students as computer-aided drawing tools have become available. The act of sketching is a both physical and mental process. A well used description of sketching is that a sketch is a designer's 'conversation with themselves'. What happens physically during sketching is easy to see and understand. The cognitive processes involved in sketching have been explored by many researchers in various concentration areas, including: engineering, architecture, art, education, and psychology. Much work has been done using protocol studies and the reader is referred to analysis of work on that topic by Purcell and Gero [6].

Sketching is not only the engineers' way to work (Ferguson, [7]) but is recognized as a tool for ideation. Larkin and Simon [8] have shown that sketches are useful in problem solving because of their conciseness of representing data compared to sentential descriptions, in part because of its spatial relationships and also because sketching allows fleeting thoughts in the mind to be captured quickly [9]. Sketches also act as gestalts allowing designers to read off from the sketch far more information that was invested initially creating the sketch. Shah et al. [10] found that the use of sketches promotes novelty and variety, two measures related to divergent thinking. McCormick [11] also asserts that "Sketching is the tool for innovation, and is so vital to the engineering process that it should be taught and used as an essential part of engineering education and professional practice." However, there is also limited evidence on the contrary. For example, Bilda et al. [12] conducted a think-aloud experiment with experienced architects $(\mathrm{N}=3)$ and reported no significant difference between sketching and not sketching during conceptual designing. Given the contradicting evidence more experimentation is necessary.

Based on the background provided above, this research work involves rigorous experimental designs to collect quantitative and qualitative data to answer the following research questions to formulate an effective curriculum for integration to design focused courses:

Research Question 1: Can TRIZ improve the ideation performance of engineering students? It is hypothesized that the training of students in the TRIZ ideation method will improve students' ability to generate a variety of innovative concepts.

Research Question 2: Can emphasizing sketching improve the ideation performance of engineering students? It is hypothesized that the emphasis on sketching will stimulate the creativity of students in design ideation.

\section{EXPERIMENTAL SETUP}

Based on the hypotheses, experiments are planned with the appropriate factors (i.e. independent variables), levels (i.e. treatments or interventions), responses (i.e. metrics), and replications (the experimentation is distributed among the participating universities (Penn State, University of Texas-El Paso, University of Maryland, and Texas A\&M University) to gather a breadth of data). The experiments are run to investigate the main effects as well as the interaction effects among factors. The data collected will be assessed using a metric system that includes Quantity, Quality, Novelty and Variety. The roadmap of our overall work is provided in Figure 1. During the experimentations, two treatments (i.e., interventions) are used. TRIZ treatment is equivalent to a basic training for the ideation method. Sketching treatment can be defined as an emphasis on the benefit of employing sketches during idea generation.

There are two fundamental values used in judging the worth of a design ideation method: how effective it is in expanding the design space and how well it explores this space. Based on that, four independent effectiveness measures are proposed for this research: quantity, quality, novelty, and variety of the ideas generated [13].

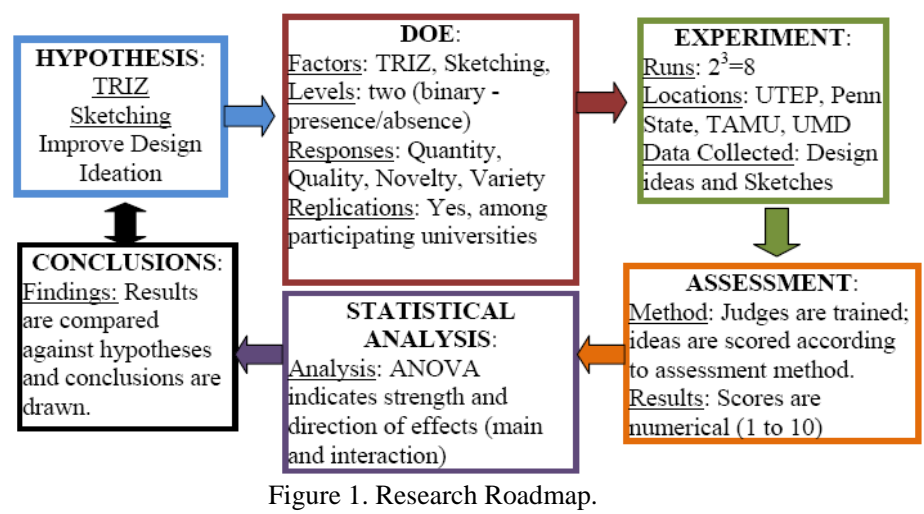




\section{A. Novelty}

Novelty is a measure of how unusual an idea is as compared to other ideas. There are two approaches to measuring Novelty: a priori and posteriori. Novelty a priori requires the predefinition of what is expected to be novel before actually analyzing the ideas. The ideas generated can be analyzed based on the functions the problem requires it to fulfill. The evaluator predefines each function at different levels (e.g. high, medium and low) based on the type of the ideas expected. The ideas falling in the corresponding level for each function receives a novelty score (e.g., High-10, Medium-5, Low-1). A novelty score for each idea can be calculated by assigning weights for each function and aggregating for an overall value. Novelty a posteriori can be calculated by counting the number of occurrences for the same idea for each function. The novelty score (S) for each function's idea can be calculated using the formula:

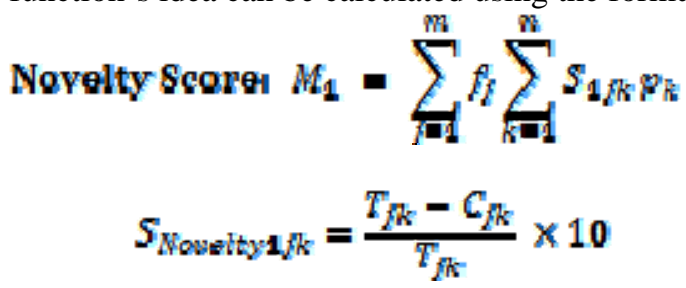

Where:

$T=$ total number of ideas for given function

$C=$ number of ocurrences of a particular solution for the given function

The higher the occurrence of a particular solution, the lower the novelty score. A novelty score for the whole idea can be calculated by assigning a weight for each function and multiplying the novelty scores for each to obtain an overall novelty score. The expression for $\mathrm{S}$ is multiplied by 10 in order to normalize it (i.e., 0 is lowest while 10 is highest).

\section{B. Variety}

Variety measures the explore solution space during the idea generation process. The uniqueness of concepts is reflected by the variety index. When calculating the variety index, concepts are rearranged into a hierarchy structure. Within a function category, all the concepts are further differentiated by their working principles. The number of working principles in a function category greatly affects the value of the relative variety index. Similar to the novelty index, the higher the value of the variety index is, the better it is. A set of ideas is analyzed for each of its functions to generate a "genealogy tree" that has at its top the function to solve, then at the next level, the physical principles (or physical effects) used. For each physical principal one or more working principles are identified, and for each working principle one or more embodiments can exist. The tree is used to calculate a Variety score for the set of ideas. More branches at higher levels of the tree means a higher variety score, while more branches at lower levels of the tree means lower variety score. The following formula calculates a score for variety:

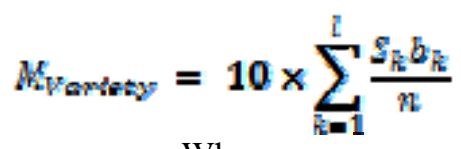

Where:

$l=$ number of levels of abstraction used to describe ideas (i.e. physical principle, working priciple, embodiment)

$S_{\mathrm{k}}=$ rating score for level $\mathrm{k}$

$b_{\mathrm{k}}=$ number of branches at level $\mathrm{k}$ where each branch represents a different component type

$n=$ number of total ideas generated in the set

Shah et al. [10]] suggest using 10, 6, 3 and 1 as $S_{\mathrm{k}}$ for the four levels structure from top. However, Nelson et al. [ $\left.{ }^{\mathrm{i}}\right]$ considered setting $S_{\mathrm{k}}$ to be $10,5,2$ and 1 for a four levels structure can provide a more differentiable result. The formula for Variety is multiplied by 10 for normalization purposes. The overall variety score is calculated by assigning weights for each function and multiplying the variety score to obtain an overall score for the set of ideas (i.e. each idea in the set has the same variety score).

Varlety Score1 $M_{3}=10 \times \sum_{i=1}^{m} f_{k} \sum_{k=1}^{i} \frac{S_{k} b_{k}}{n}$

C. Quantity

Quantity is the total number of ideas generated in a specified amount of time. The premise of this measure is that generating more ideas increases the chance of better ideas. This score is directly assigned by counting the number of ideas each subject records during an experiment.

\section{PILOT EXPERIMENTATION RESULTS \& DISCUSSION}

The complexity of this study led the researchers to pilot the experiment in some of the treatment conditions and in selected design courses. Each University followed a different procedure for the pilot study. This allowed us to explore different ways to conduct experiments and helped us gain a varied experience that will be useful to define a unified design of experiment for the four universities. In this paper, we are only revealing the results of the pilot study for the Penn State site. Across data collection locations, however, the same ideation task was used, which is provided as: "In rural areas of developing countries, such as Kenya, cooking is done in the home with biomass type cooking systems. One of the adverse affects of these cooking systems is the emissions which cause respiratory illnesses for millions of children and women. The people in these developing countries are economically and culturally constrained by the types of cooking systems they use. Also, depending on the type of biomass used there can be unsustainable and detrimental effects on the environment. Develop several concepts for a cooking system that is culturally appropriate, sustainable and low cost to meet the needs of rural Kenya."

At Penn State, data collection was done in two different courses: EDGSN 100, and QMM492. Four sections of the 
EDSGN 100 course were involved in data collection yielding over 120 individual data points and 32 team level data sets. Out of these sections, three of them were taught by the same instructor. In two sections (where TRIZ training was provided), instructors were different, and the level of the bias introduced due to having different instructors was intended to be measured. In QMM492, a senior course for nonengineering students, data was collected to be compared to senior engineering students to understand the importance of the background knowledge of students.

For the two courses, Introduction to Engineering Design (EDSGN 100) (four course sections), and Engineering Design Principles (QMM 492) (one course section), we collected all concepts generated by the students for the Ideation Task, and organized them into three functional categories: Energy Source \& Fuel, Function and Body Design. We used three indices, the novelty index, the variety index and the quantity, which were described above, to examine ideation performance of the concepts. We present the results at the functional level (not in synthesized designs) below.

Below we illustrate the computation process by using a real example from our dataset. Table 1 is the energy source \& fuel category from the Sketch class. The fuel function includes 13 concepts related to the way of generating energy. In the Sketch class, students generated a total of 27 ideas related to all the 13 concepts. Thus, $\mathrm{C}_{i}=27$. The calculation of $\mathrm{Si}$ is provided below.

Take A1, the solar energy concept as an example. The S value for A1 is 7.40740. To aggregate the novelty index for fuel function, we multiply Ci with Si for each concept and sum them up. Then, we divide the obtained sum by the number of concepts $n$, where $n=13$ for the energy source \& fuel function. Table 2 presents a summary of the data from the pilot study at Penn State.

$$
S_{1}=\left(\frac{27-7}{27}\right) \times 10=7.407407
$$

TABLE I. NOVELTY INDEX TABLE FOR THE SKETCH CLASS

\begin{tabular}{|c|l|c|c|}
\hline & Concepts in the Design Space & C & S \\
\hline A1 & Solar energy & 7 & 7.4 \\
\hline A2 & Human energy (Crank generator) & 1 & 9.6 \\
\hline A3 & Wood & 4 & 8.5 \\
\hline A4 & Wind & 0 & 10 \\
\hline A5 & Alcohol & 0 & 10 \\
\hline A6 & Chemical & 0 & 10 \\
\hline A7 & Dung & 0 & 10 \\
\hline A8 & Natural gas & 3 & 8.89 \\
\hline A9 & Electricity & 6 & 7.78 \\
\hline A10 & Fuel & 4 & 8.51 \\
\hline A11 & Biomass & 1 & 9.6 \\
\hline A12 & Battery powered & 0 & 10 \\
\hline A13 & Mirror reflect & Novelty & $\mathbf{1 7 . 0 9}$ \\
\hline & & & \\
\hline
\end{tabular}

For the calculation of the variety index, generated ideas for the energy source and fuel function are put into a hierarchy. For the above example, there are two levels, three working principles and eight effective concepts. Thus, for this example, we have $\mathrm{p}=2, B_{1}=3, B_{1}=8$, and $n=13$. Since the sum of $f_{\mathrm{i}}$ equals 1 , we can have the variety index for this fuel function as $\mathrm{M}=1 *(5 * 3+2 * 9) / 13=2.38$.

The rest of the metrics for the design ideas generated by the Penn State students are calculated as presented in Table 2. In the table, the total generated ideas, as well as total generated unique ideas by class section are also reported. Upon review of the data presented in Table 2, one would see that overall Sketch and Control sections (as labeled in column headers) seem to have performed better in terms of novelty and quantity scores. For the other metrics, differences across section results seem to narrow. However, these results do not take into account the semester standing of the engineering students who participated in the study (i.e., freshman, sophomore, etc.).

In order to understand the potential impact of the semester standing of the participants in the generated results, we have plotted the novelty, variety, and quantity scores across the average semester standing value for the class section. The average semester standing value for each class section is operationalized as the weighted average of the sumof the products of number of students across semester standing levels (e.g., ((3 students $* 1^{\text {st }}$ semester $)+2$ students $* 2^{\text {nd }}$ semester $\left.)\right) / 5$ students).

Figures 2 and 3 provide the plots of novelty and variety values versus the semester standing. As seen below, as with the increasing semester standing while the novelty scores get lower, variety scores are steady across class sections with increasing average weighted semester standing values. A similar observation is also made for the quantity values in that when the total number of ideas generated is reported, with the increasing semester standing the values get smaller, and when only the unique ideas are reported, the metric values stay steady.

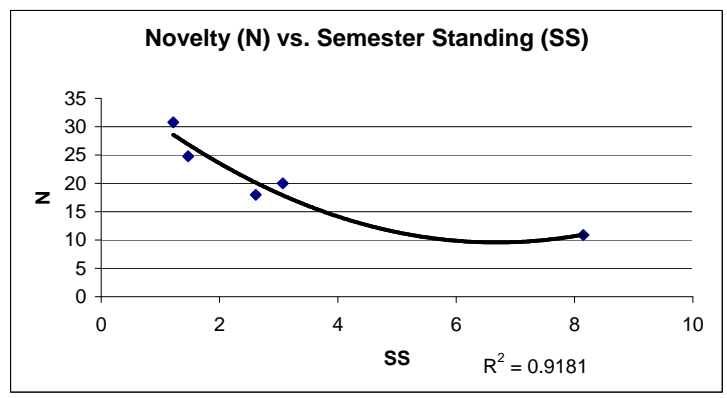

Figure 2. Novelty Metric Scores. 


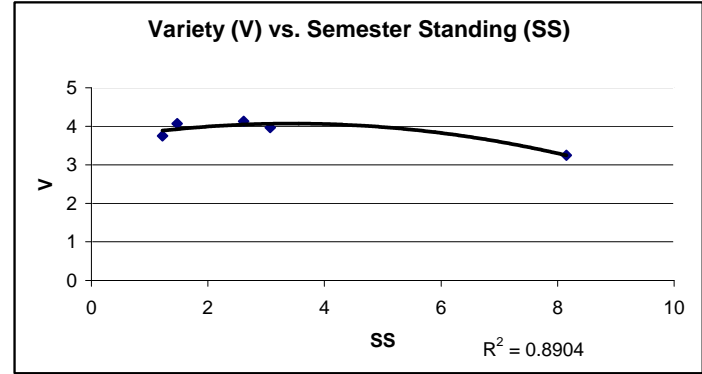

Figure 3. Variety Metric Scores.

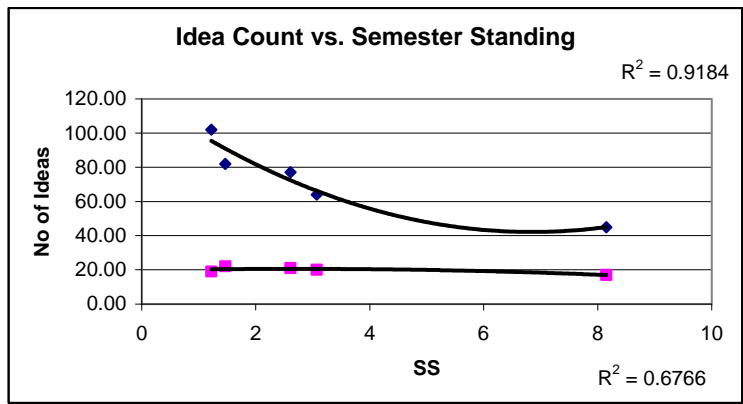

Figure 4. Total Ideas and Total Unique Ideas.

Regardless of the treatment conditions of the class sections, we speculate that there are meaningful reasons for the drop in novelty and the total number of ideas generated, and the steady level for variety and the total number of unique ideas. For example, one might speculate that perhaps engineering students' ideation fluency could be impacted adversely by their education as they progress in their curriculum. To this end, studies have documented (1) that people whose personality types indicate high levels of creative potential are leaving engineering at higher rates than the student body average, and (2) faculty teaching methods lean heavily towards a 'plug-and-chug' approach to engineering problem solving, stifling creativity [14]. Indeed, one of the earliest accounts of this is by A.D. Moore [15] - an engineering professor - : "I wish I could say that these educational areas [science and engineering] also have, as a main purpose, the stimulation of your creativity, and that they succeed in doing it. I am afraid that neither is true. In fact, I suspect that the taking of a degree in engineering or science may, in many cases, do more to stifle creativity than to stimulate it.” Indeed, the situation of slightly increasing design knowledge (and semester standing) but considerably decreasing originality and fluency of ideas generated as measured by a subtask of Torrance test has been reported [16].

However, the above stated reason does not explain the steady the levels in the reported variety metrics or the total unique idea counts. Perhaps, what might happen is that as with the increasing semester standing, students learn to efficiently evaluate, and accept or reject the ideas while they generate ideas given a design problem solving context. However, as part of the pilot studies conducted, we have not gathered information on students' ideation thinking, and thus cannot ascertain the reasons.
Overall, these observations reflect the complexity of collecting data engineering ideation data in classroom settings. These observations will be used to better design the follow up experiments.

\section{ACKNOWLEDGMENT}

The authors gratefully acknowledge the support of the National Science Foundation grant. The project is funded by NSF grant CCLI-II 0920446. The opinions expressed in this paper do not necessarily reflect those of NSF. The authors also acknowledge the student participants in the pilot studies.

\section{REFERENCES}

[1] Okudan, G.E., Ogot, M. and Shirwaiker, R. "An Investigation on the Effectiveness Design Ideation Using TRIZ," Proceedings of IDETC/CIE 2006 Design Education Conference, September 10-13, 2006, Philadelphia, PA.

[2] Shah, J., Vargas-Hernandez, H., and Smith, S. (2003). "Metrics for Measuring Ideation Effectiveness." Design Studies, 24(2), 111-134.

[3] Howard, Thomas, Culley, Steve, Dekoninck, "Creative Stimulations in Conceptual Design: An Analysis of Industrical Case Studies," DETC2008-49672, Proceedings of the ASME 2008 International Design Engineering Technical Conferences \& Computers in Engineering Conference, August 3-6, 2008, Brooklyn, New York, USA, 2008.

[4] Tseng, Ian, Moss, Jarrod, Cagan, Jonathan, Kotovsky, Kenneth, "Overcoming Blocks in Conceptual Design: The effects of Open Goals and Analogical Similarity on Idea Generation," DETC2008-49276, Proceedings of the ASME 2008 International Design Engineering Technical Conferences \& Computers in Engineering Conference, August 3-6, 2008, Brooklyn, New York, USA, 2008.

[5] Chiu, I, Shu, L.H., "Effects of Dichotomous Lexical Stimuli in Concept Generation," DETC2008-49372, Proceedings of the ASME 2008 International Design Engineering Technical Conferences \& Computers in Engineering Conference, August 3-6, 2008, Brooklyn, New York, USA, 2008.

[6] Purcell, A. T., and Gero, J. S., (1998) "Drawings and the design process: A review of protocol studies in design and other disciplines and related research in cognitive psychology,” Design Studies, Volume 19, Issue 4, Pages 389-430.

[7] Ferguson, E. (1992), Engineering and the Mind's Eye, The MIT Press, Cambridge.

[8] Larkin and Simon (1987), "Why a Diagram is (Sometimes) Worth Ten Thousand Words”, Cognitive Science, Col. 11, pp. 65-99.

[9] Hanks and Belliston, 1980, Rapid Viz: A New Method for the Rapid Visualization of Ideas, Kaufmann, Los Altos, CA.

[10] Shah, J., Vargas-Hernandez, N., Summers, J., and Kulkarni, S., (2001), "Collaborative Sketching (C-Sketch): An Idea Generation Technique for Engineering Design", Journal of Creative Behavior, 35(3), pp. 168-198.

[11] McCormick, D. (2007). "Seeing Mechanical." Mechanical Engineering, 35-36.

[12] Bilda, Z., Gero, J., and Purcell, T. (2006). "To Sketch or Not to Sketch? That is the Question.”Design studies, 27, 587-613.

[13] Nelson, B. A., Yen, Jeannettte, Wilson, J. O., and Rosen, D., (2009), "Refined metrics for measuring ideation effectiveness", Design Studies, 30, pp. 737-743.

[14] Lumsdaine, M. and Lumsdaine, E., "Thinking Preferences of Engineering Students: Implications for Curriculum Restructuring." Journal of Engineering Education, Vol. 84, No. 2, 1995, pp. 194-204.

[15] Moore, A.D., Invention, Discovery, and Creativity, Anchor Books, New York: Doubleday \& Company, Inc., 1969.

[16] Christine Beisler Masters, Mieke Schuurman, Gul Okudan, and Samuel T Hunter (2008) "An Investigation of Gaps in Design Process Learning: Is there a Missing Link Between Breadth and Depth,” in proceedings American Society for Engineering Education Annual Conference \& Exposition Proceedings, American Society for Engineering Education Annual Conference \& Exposition Proceedings. 
TABLE 1 SUMMARY OF PILOT DATA

\begin{tabular}{|c|c|c|c|c|c|}
\hline \begin{tabular}{|l|} 
Experimental Condition \\
\end{tabular} & Sketch Emphasis & Control Group & TRIZ & TRIZ & TRIZ \\
\hline Course & EDSGN 100 - Section 11 & EDSGN 100 - Section 15 & EDSGN 100 - Section 6 & EDSGN 100 - Section 16 & QMM 492 \\
\hline Function & \multicolumn{5}{|c|}{ NOVELTY METRIC VALUES } \\
\hline Energy Source \& Fuel & 17.09 & 19.23 & 15.00 & 14.46 & 13.07 \\
\hline Functionality & 32.64 & 43.15 & 22.86 & 16.30 & 5.14 \\
\hline Body Design & 42.52 & 11.95 & 22.14 & 23.35 & 14.43 \\
\hline $\begin{array}{l}\text { Average Metric Value } \\
\text { Across Functions }\end{array}$ & 30.75 & 24.78 & 20.00 & 18.04 & 10.88 \\
\hline Function & \multicolumn{5}{|c|}{ VARIETY METRIC VALUES } \\
\hline Energy Source \& Fuel & 2.38 & 3.08 & 2.77 & 2.54 & 3.46 \\
\hline Functionality & 4.57 & 4.57 & 4.57 & 5.29 & 3.29 \\
\hline Body Design & 4.29 & 4.57 & 4.57 & 4.57 & 3.00 \\
\hline $\begin{array}{l}\text { Average Metric Value } \\
\text { Across Functions }\end{array}$ & 3.75 & 4.07 & 3.97 & 4.13 & 3.25 \\
\hline Function & \multicolumn{5}{|c|}{ QUANTITY METRIC VALUES } \\
\hline Energy Source \& Fuel & 27.00 & 32.00 & 24.00 & 25.00 & 20.00 \\
\hline Functionality & 33.00 & 39.00 & 20.00 & 17.00 & 5.00 \\
\hline Body Design & 42.00 & 11.00 & 20.00 & 35.00 & 20.00 \\
\hline $\begin{array}{l}\text { Total Metric Value } \\
\text { Across Functions }\end{array}$ & 102.00 & 82.00 & 64.00 & 77.00 & 45.00 \\
\hline Function & \multicolumn{5}{|c|}{ UNIQUE IDEA COUNT } \\
\hline Energy Source \& Fuel & 8.00 & 10.00 & 8.00 & 9.00 & 10.00 \\
\hline Functionality & 6.00 & 6.00 & 6.00 & 6.00 & 4.00 \\
\hline Body Design & 5.00 & 6.00 & 6.00 & 6.00 & 3.00 \\
\hline $\begin{array}{l}\text { Total Metric Value } \\
\text { Across Functions }\end{array}$ & 19.00 & 22.00 & 20.00 & 21.00 & 17.00 \\
\hline
\end{tabular}

